\title{
Associations between serum uric acid levels and the incidence of hypertension and metabolic syndrome: a 4-year follow-up study of a large screened cohort in Okinawa, Japan
}

\author{
Kazufumi Nagahama $^{1}$, Taku Inoue ${ }^{2}$, Kentaro Kohagura ${ }^{1}$, Kozen Kinjo ${ }^{3}$ and Yusuke Ohya ${ }^{1}$
}

The purpose of this study was to examine the associations between serum uric acid (SUA) levels and the incidences of hypertension and metabolic syndrome (MetS) in a large screened cohort of Japanese men and women. We evaluated 4812 subjects (males, 2528; females, 2284; mean age, 47.5 years) who underwent health checkups between 2006 and 2010 and were free of hypertension and MetS in 2006. After 4 years, $618(13 \%), 764(16 \%)$ and $158(3 \%)$ subjects developed hypertension, MetS and hypertension with MetS, respectively. Increased SUA levels were significantly and positively associated with the incidences of hypertension, MetS and hypertension with MetS. Compared with the first quartile of SUA levels, the odds ratios $(95 \%$ confidence intervals) for the third and fourth quartiles, respectively, were as follows: $1.5(1.1-2.1 ; P=0.0128)$ and $1.8(1.2-2.5 ; P=0.0022)$ for hypertension, $1.3(0.9-1.9 ; P=0.1910)$ and $1.8(1.2-2.7 ; P=0.0039)$ for MetS and 2.7 (1.1-6.6; $P=0.0276)$ and 3.2 (1.3-8.0; $P=0.0115)$ for hypertension with MetS. In conclusion, increased SUA levels were significantly and independently associated with the incidences of hypertension and MetS in subjects without hypertension or MetS at baseline. Increased SUA levels might also be correlated with the incidence of hypertension with MetS. Hypertension Research (2015) 38, 213-218; doi:10.1038/hr.2014.161; published online 6 November 2014

Keywords: cardiovascular risk factors; cohort study; epidemiology; metabolic syndrome; uric acid

\section{INTRODUCTION}

Elevated serum uric acid (SUA) levels are closely associated with hypertension and metabolic syndrome (MetS). MetS is characterized by the presence of multiple common cardiovascular risk factors, such as central obesity, atherogenic dyslipidemia (hypertriglyceridemia and low high-density lipoprotein cholesterol (HDL-C)) levels, hyperglycemia and elevated blood pressure (BP). Elevated SUA levels are prevalent in patients with hypertension ${ }^{1,2}$ and MetS, ${ }^{3-7}$ and these levels can be a significant predictor of the development of hypertension ${ }^{3,8-10}$ and MetS. ${ }^{11-14}$ Although evidence has suggested that elevated SUA levels might have a role in hypertension, ${ }^{15}$ a causal relationship has not been established. Confirming the involvement of elevated SUA levels in the pathogenesis of hypertension has been difficult, because MetS can confound the relationship between elevated SUA levels and hypertension and because these conditions share common pathophysiological features, such as insulin resistance, endothelial dysfunction, oxidative stressand inflammation. ${ }^{16-18}$ For these reasons, controlling for MetS is important in clinical studies that examine the association between elevated SUA levels and the incidence of hypertension.

We recently reported that hyperuricemia was a significant and independent predictor of MetS. ${ }^{11}$ In that study, the prevalence of hypertension was significantly higher in women who developed MetS than in those who did not. Substantial evidence has also indicated a higher prevalence of insulin resistance among patients with hypertension than among normotensive individuals. ${ }^{19-21}$ These findings have also given rise to the clinical question of whether elevated SUA levels are associated with incident MetS (also called insulin resistance syndrome) in subjects without hypertension.

In subjects with hypertension, MetS was independently associated with insufficient BP control and lower effectiveness of antihypertensive therapy. ${ }^{22}$ Moreover, Ninomiya et al. ${ }^{23}$ showed that, among middleaged Japanese men and women, hypertensive subjects with MetS had a significantly higher risk of cardiovascular disease, compared with those without MetS. In this study, the risk of cardiovascular disease in normotensive subjects with MetS was similar to that in hypertensive

\footnotetext{
${ }^{1}$ Department of Cardiovascular Medicine, Nephrology and Neurology, Graduate School of Medicine, University of the Ryukyus, Okinawa, Japan; ${ }^{2}$ Center of Residency and Fellowship Program, Graduate School of Medicine, University of the Ryukyus Hospital, Okinawa, Japan and ${ }^{3}$ The Okinawa General Health Association, Okinawa, Japan Correspondence: Dr K Nagahama, Department of Cardiovascular Medicine, Nephrology and Neurology, Graduate School of Medicine, University of the Ryukyus, 207 Uehara, Nishiharacho, Okinawa 903-0125, Japan.

E-mail: orch2970@med.u-ryukyu.ac.jp

Received 7 November 2013; revised 27 August 2014; accepted 3 September 2014; published online 6 November 2014
} 
subjects without MetS. Therefore, hypertension with MetS might confer a high risk of cardiovascular events.

The purpose of this study was to examine the associations between SUA levels and the incidence of hypertension, MetS and hypertension with MetS in subjects without hypertension or MetS in a large screened cohort of Japanese men and women.

\section{METHODS}

\section{Subjects}

In 2006, 13668 individuals participated in a 1-day health checkup conducted by the Okinawa General Health Association. Of these participants, 6919 were re-examined in 2010; from this cohort, we excluded 17 participants because of a lack of available data on waist circumference (WC), as well as 1124 participants with hypertension, 314 with MetS and 652 with MetS in 2006. A total of 4812 individuals (male, 2528; female, 2284) were thus included in this study cohort. This study was approved by the ethics committee of the University Hospital of the Ryukyus.

The Okinawa General Health Association conducted the health checkups in Okinawa, Japan. Subjects who visited the clinic of the Okinawa General Health Association completed a questionnaire that included details on the following factors: family history of hypertension, diabetes mellitus, dyslipidemia, stroke and heart disease; lifestyle-related variables such as smoking and alcohol intake; medical history; and current medications. The participants were classified into one of the following three categories based on their smoking habits: nonsmokers, ex-smokers, or current smokers. Regarding alcohol intake, subjects were classified as non-drinkers, ex-drinkers or current drinkers. Current medications included the use of antihypertensive medications (yes/no), antidiabetic medications and/or insulin (yes/no) and antidyslipidemic medications (yes/no). The questionnaires were discussed during the physical examinations, and the subjects were further interviewed by a clinic physician.

All the subjects underwent clinical examinations that included height, weight and WC measurements. WC was measured using a graduated tape at the umbilical level with the subject in the standing position. Body mass index (BMI) was calculated as body weight $(\mathrm{kg})$ divided by height squared $\left(\mathrm{m}^{2}\right)$. A well-trained nurse or doctor measured the resting BP once using a standard mercury sphygmomanometer (KENZMEDICO, Saitama, Japan) with the subject in the sitting position after at least 5 min of rest.

All the subjects fasted overnight for at least $12 \mathrm{~h}$ before blood sampling. A venous blood sample was obtained from the antecubital vein. SUA, triglycerides (TGs), HDL-C and fasting blood glucose (FBG) levels were determined for all the subjects using an autoanalyzer (Hitachi Automatic Analyzer Model 7700, Hitachi High-Technologies, Tokyo, Japan) in the laboratory of the Okinawa General Health Association.

\section{Definitions}

There is no universally accepted definition of hyperuricemia. In the present study, we defined hyperuricemia as SUA levels $>7.0 \mathrm{mg} \mathrm{dl}^{-1}$ according to the Japanese criteria for hyperuricemia. ${ }^{24}$ SUA levels were categorized by quartiles as follows: $\mathrm{UA} 1, \leqslant 4.4 \mathrm{mg} \mathrm{dl}^{-1}$; UA2, 4.5-5.4 $\mathrm{mg} \mathrm{dl}^{-1}$; UA3, $5.5-6.4 \mathrm{mg} \mathrm{dl}^{-1}$; and $\mathrm{UA} 4, \geqslant 6.5 \mathrm{mg} \mathrm{dl}^{-1}$. In the subgroup analyses of men and women, SUA levels were categorized into three groups according to tertile: $\mathrm{UA} 1, \leqslant 5.8 \mathrm{mg} \mathrm{dl}^{-1}$; UA2, $5.9-6.8 \mathrm{mg} \mathrm{dl}^{-1}$; and $\mathrm{UA} 3, \geqslant 6.9 \mathrm{mg} \mathrm{dl}^{-1}$ for the men and $\mathrm{UA} 1, \leqslant 4.1 \mathrm{mg} \mathrm{dl}^{-1}$; UA2, $4.2-4.9 \mathrm{mg} \mathrm{dl}^{-1}$; and UA3, $\geqslant 5.0 \mathrm{mg} \mathrm{dl}^{-1}$ for the women. Hypertension was defined as a systolic blood pressure (SBP) of $\geqslant 140 \mathrm{~mm} \mathrm{Hg}$ and/or a diastolic blood pressure (DBP) of $\geqslant 90 \mathrm{~mm} \mathrm{Hg}$ and/or the current use of antihypertensive medication. Obesity was defined as a BMI of $\geqslant 25 \mathrm{~kg} \mathrm{~m}^{-2}$. The estimated glomerular filtration rate (eGFR) was calculated using the modified Cockcroft-Gault formula for Japanese populations. ${ }^{25}$

For this study, MetS was defined according to the Japanese Committee on the Criteria for MetS, ${ }^{26}$ which are commonly used in routine clinical practice in Japan. Subjects with an increased WC ( $\geqslant 85 \mathrm{~cm}$ in men and $\geqslant 90 \mathrm{~cm}$ in women) and those with $\geqslant 2$ of the following factors were diagnosed with MetS: high BP $(\mathrm{SBP} \geqslant 130 \mathrm{~mm} \mathrm{Hg}, \mathrm{DBP} \geqslant 85 \mathrm{~mm} \mathrm{Hg}$ and/or the current use of antihypertensive medications); dyslipidemia (TG levels $\geqslant 150 \mathrm{mg} \mathrm{dl}^{-1}$, HDL-C levels $<40 \mathrm{mg} \mathrm{dl}^{-1}$ and/or the current use of antidyslipidemic medications) or high blood glucose (FBG levels $\geqslant 110 \mathrm{mg} \mathrm{dl}^{-1}$ and/or the current use of antidiabetic medications).

Table 1 Baseline characteristics of subjects according to serum uric acid quartiles

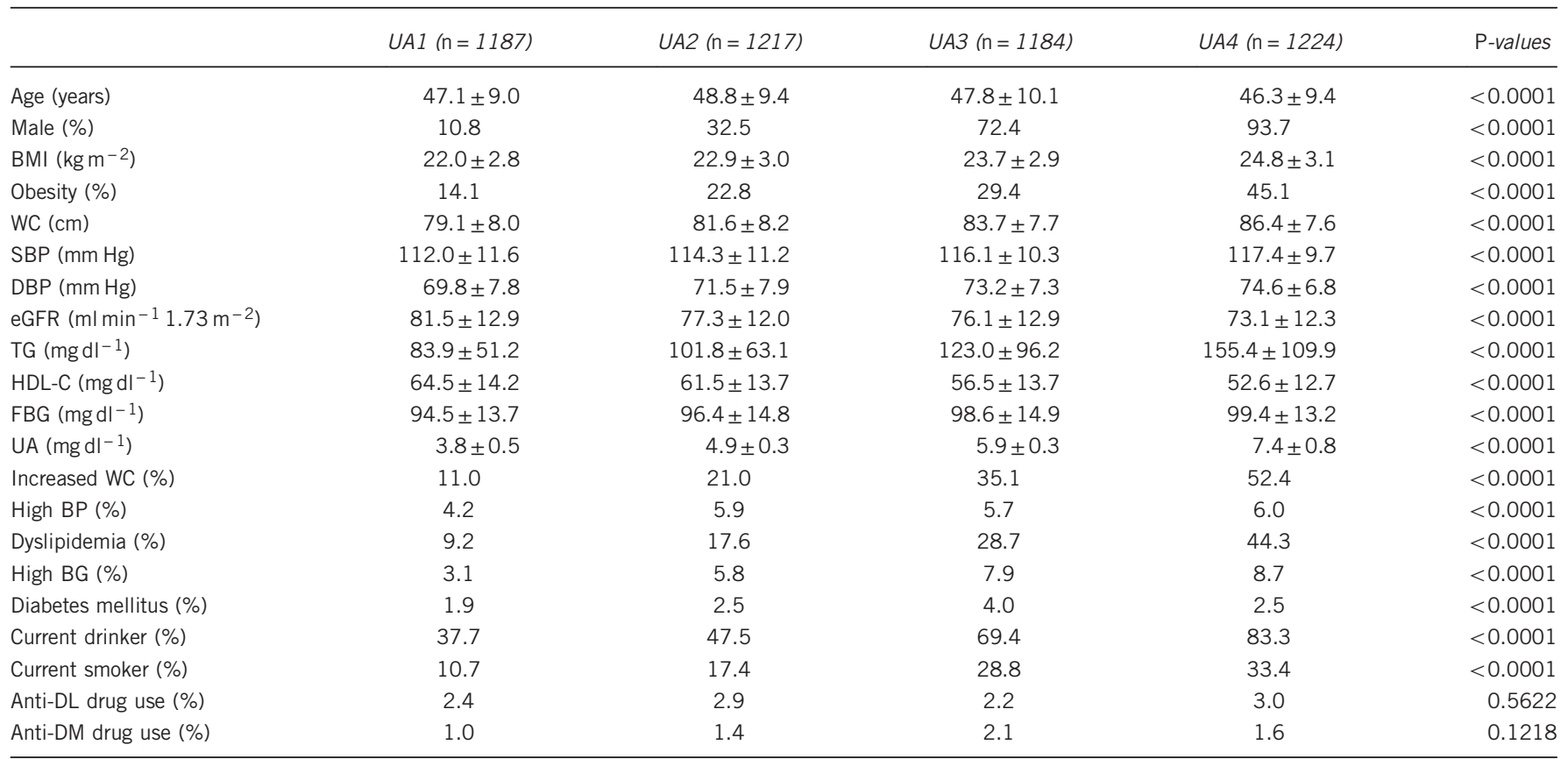

Abbreviations: Anti-DL, antidyslipidemic; Anti-DM, antidiabetic; Anti-HT, antihypertensive; BMI, body mass index; DBP, diastolic blood pressure; FBG, fasting blood glucose; HDL-C, high-density lipoprotein cholesterol; HU, hyperuricemia; SBP, systolic blood pressure; TG, triglyceride; UA, uric acid; UA1, $\leqslant 4.4 \mathrm{mg} \mathrm{dl}^{-1}$; UA2, 4.5-5.4 mg dl ${ }^{-1}$; UA3, $5.5-6.4 \mathrm{mg} \mathrm{dl}^{-1}$; UA4, $\geqslant 6.5 \mathrm{mg} \mathrm{dl}^{-1}$;

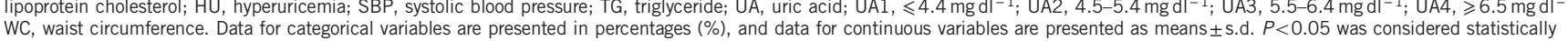
significant. 


\section{Statistical analyses}

The data for continuous variables are reported as the means \pm s.d., and the data for categorical variables are reported as percentages (\%). Independent $t$-tests were used to compare the results for continuous variables between groups with and without hyperuricemia. Data for the categorical variables were compared using the chi-square test. Odds ratios (ORs, 95\% confidence intervals (CIs)) for the incidence of MetS were determined using multivariate logistic regression models. We used two logistic models: Model 1 (adjusted for age and sex) and Model 2 (adjusted for age, sex, alcohol consumption, eGFR, WC values and SBP, TG, HDL-C, low-density lipoprotein cholesterol and FBG levels). The TG levels were transformed logarithmically to improve skewing before analyses. The lifestyle factors and variables that were correlates with hypertension, MetS and UA levels were selected as adjustment variables. The statistical analyses were performed using the JMP software, version 10.0 (SAS Institute, Cary, NC, USA). $P<0.05$ was considered statistically significant.

\section{RESULTS}

At baseline, the mean age of the subjects was $47.5 \pm 9.5$ years, and $52.5 \%$ were male. The baseline characteristics of the subjects according to the SUA quartiles are shown in Table 1. BMI and WC values, SBP, DBP, TG and FBG levels, and the prevalence of male sex, obesity, dyslipidemia, current drinking and smoking significantly increased with increasing SUA levels. In contrast, eGFR and HDL-C levels significantly decreased with increasing SUA levels.

In 2010, 618 subjects (13\%) had developed hypertension, and 764 (16\%) had developed MetS. Hypertension with MetS was found in 158 subjects (3\%). Figure 1 shows the incidences of hypertension, MetS

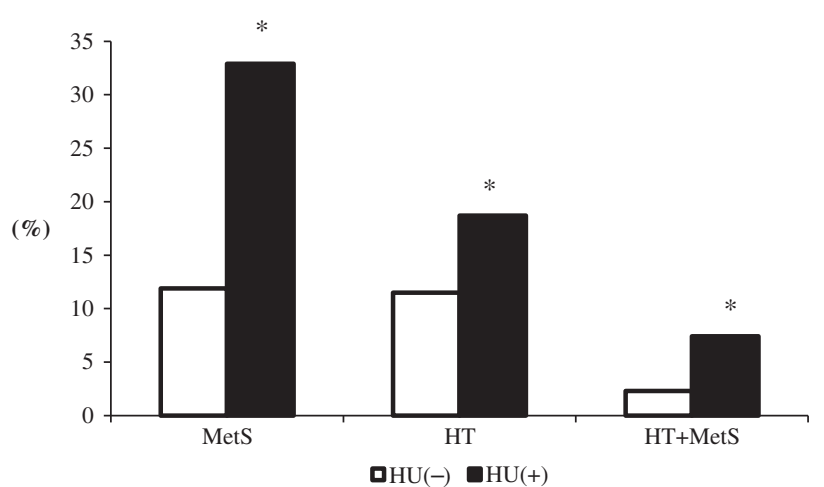

Figure 1 Incidences of hypertension, metabolic syndrome and hypertension with metabolic syndrome with and without hyperuricemia. MetS, metabolic syndrome; HT, hypertension; HT+MetS, hypertension with metabolic syndrome; $\mathrm{HU}$, hyperuricemia. ${ }^{*} P<0.05$ compared with the $\mathrm{HU}(-)$ group using the chi-square test. and hypertension with MetS in subjects with and without hyperuricemia. The incidences of hypertension, MetS and hypertension with MetS were significantly higher in subjects with hyperuricemia than in those without. ORs (95\% CIs) for hyperuricemia and for the incidences of hypertension, MetS and hypertension with MetS are shown in Table 2. Hyperuricemia was significantly associated with the incidence of both hypertension and MetS in both Model 1 and Model 2. In contrast, no significant association was found in the incidence of hypertension with MetS in Model 2.

Figure 2 shows the incidences of hypertension, MetS (Figure 2a) and hypertension with MetS (Figure 2b) according to SUA quartiles. The incidences of hypertension, MetS and hypertension with MetS significantly increased with increasing SUA levels.

ORs (95\% CIs) for the incidences of hypertension, MetS and hypertension with MetS in the second, third and fourth SUA quartiles, compared with that in the first SUA quartile, are shown in Table 3. In Model 1, the third and fourth quartiles were significantly associated with the incidences of hypertension, MetS and hypertension with MetS. In Model 2, the fourth quartile was significantly associated with the incidences of hypertension, MetS and hypertension with MetS. The analyses were repeated using SUA levels as a continuous variable. Significant associations were observed between increased SUA levels $\left(1 \mathrm{mg} \mathrm{dl}^{-1}\right)$ and the risk of hypertension, MetS and hypertension with MetS in Model 1 (hypertension: OR, 1.2; 95\% CI, 1.1-1.3; $P<0.0001$; MetS: OR, 1.5; 95\% CI, 1.4-1.7; $P<0.0001$; hypertension with MetS: OR, 1.5; 95\% CI, 1.4-1.8; $P<0.0001$ ) and Model 2 (hypertension: OR, $1.1 ; 95 \%$ CI, $1.0-1.2 ; P=0.0073$; MetS: OR, $1.2 ; 95 \%$ CI, $1.1-1.3$; $P<0.0001$; hypertension with MetS: OR, 1.3; 95\% CI, 1.1-1.5; $P=0.0002$ ).

Because SUA levels differed by sex, we conducted subgroup analyses of men and women. Table 4 shows the incidences of hypertension, MetS and hypertension with MetS according to the presence of hyperuricemia and the UA tertile in men and women. Female subjects with hyperuricemia accounted for a small fraction of the incidences of hypertension, MetS and hypertension with MetS. In Model 2 (Table 4), male subjects with hyperuricemia had significant associations with the incidences of both hypertension and MetS. Compared with the first SUA tertile, the third tertile was significantly associated with the incidences of MetS and hypertension with MetS only in men. In contrast, in women, hyperuricemia had no significant association with the incidences of hypertension or MetS (Table 4).

\section{DISCUSSION}

In this 4-year follow-up study, we observed that elevated SUA levels were significantly and independently associated with the incidences of

Table 2 Odds ratios for the incidence of hypertension, metabolic syndrome and hypertension with metabolic syndrome according to the presence or absence of hyperuricemia

\begin{tabular}{|c|c|c|c|c|c|c|c|c|}
\hline & \multicolumn{2}{|c|}{ Incidence (n, \%) } & \multicolumn{3}{|c|}{ Model 1} & \multicolumn{3}{|c|}{ Model 2} \\
\hline & $H U(-)(n=4131)$ & $H U(+)(n=681)$ & $O R$ & $95 \% \mathrm{Cl}$ & P-values & $O R$ & $95 \% \mathrm{Cl}$ & P-values \\
\hline MetS & 515 (12.5) & 249 (36.6) & 2.3 & $1.9-2.7$ & $<0.0001$ & 1.3 & $1.0-1.6$ & 0.0217 \\
\hline HT+MetS & $105(2.5)$ & $53(7.8)$ & 2.0 & $1.4-2.8$ & 0.0002 & 1.4 & $0.9-2.0$ & 0.1117 \\
\hline
\end{tabular}

Abbreviations: $\mathrm{Cl}$, confidence interval; $\mathrm{HT}$, hypertension; $\mathrm{HT}+$ MetS, hypertension with metabolic syndrome; MetS, metabolic syndrome; OR, odds ratio.

Model 1: adjusted for age and sex.

Model 2: adjusted for age, sex, alcohol consumption, estimated glomerular filtration rate, waist circumference values and systolic blood pressure, triglyceride, high-density lipoprotein cholesterol, low-density lipoprotein cholesterol and fasting blood glucose levels

Hyperuricemia was defined as serum uric acid levels of $>7.0 \mathrm{mg} \mathrm{dl}^{-1}$

$P<0.05$ was considered statistically significant. 


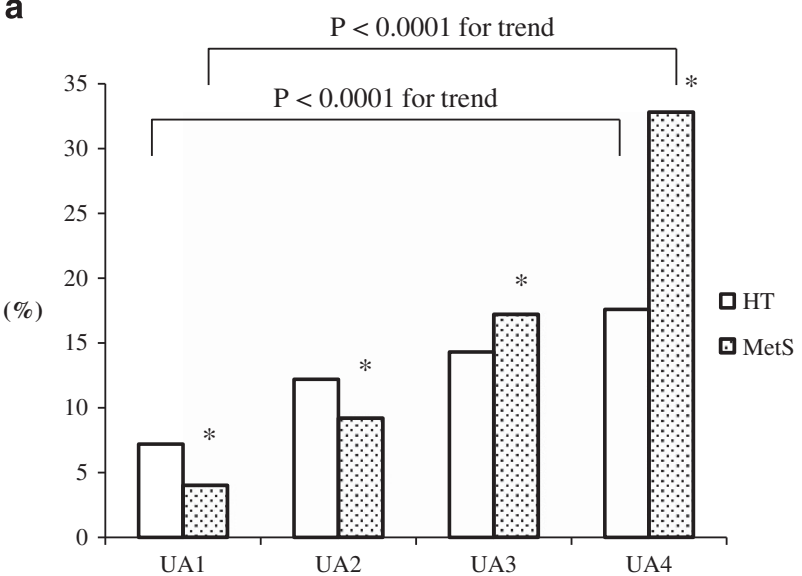

b

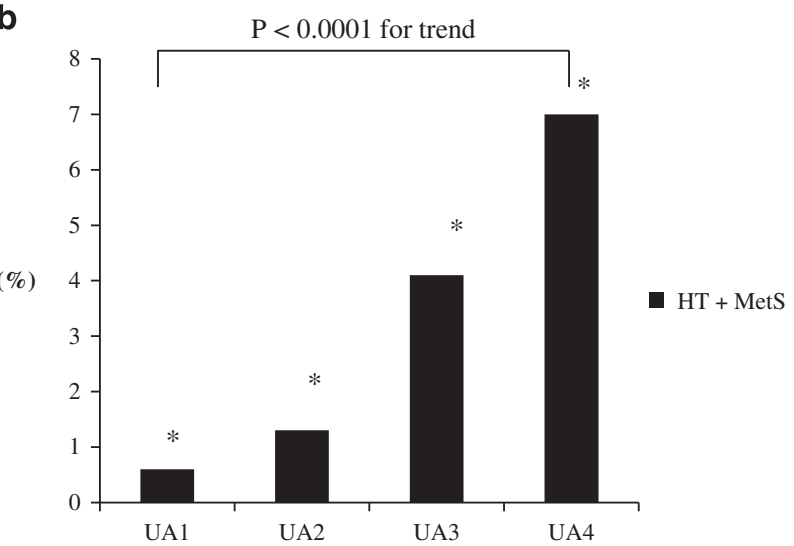

Figure 2 (a and $\mathbf{b}$ ) Incidences of hypertension and metabolic syndrome (a) and of hypertension with MetS (b) according to serum uric acid quartiles. MetS, metabolic syndrome; HT, hypertension; HT+MetS, hypertension with metabolic syndrome. UA, uric acid; UA1, $<4.5 \mathrm{mg} \mathrm{dl}^{-1}$; UA2, 4.5$5.4 \mathrm{mg} \mathrm{dl}^{-1} ; \quad$ UA3, $\quad 5.5-6.4 \mathrm{mg} \mathrm{dl}^{-1} ; \quad$ UA4, $\geqslant 6.5 \mathrm{mg} \mathrm{dl}^{-1} . \quad * P<0.05$ compared with the $\mathrm{HT}(+)$ group using the chi-square test. hypertension, MetS and hypertension with MetS in subjects who did not have hypertension or MetS at baseline. These associations were independent of age, sex, lifestyle variables, MetS-component levels and renal function. In the subgroup analysis, hyperuricemia showed significant associations with the incidences of both hypertension and MetS in males.

The Multiple Risk Factor Intervention Trial (MRFIT) study recently investigated the effect of hyperuricemia on the risk of hypertension in normotensive men without MetS. ${ }^{10}$ In that study, subjects with hyperuricemia were at an $80 \%$ higher risk of developing hypertension, compared with those without hyperuricemia. The MRFIT study was a randomized, controlled trial in a cohort of men who were at high risk for adverse coronary events. In contrast, our study included both male and female subjects in good health. Despite differences in some of the results between these two studies, both studies found that hyperuricemia was significantly associated with the incidence of hypertension in men who did not have MetS at baseline. No significant associations were found between hyperuricemia and the incidences of hypertension or MetS in women in our study. One reason for this result might have been the small number of female participants who developed hypertension or MetS in this study, resulting in low statistical power for female subjects (Table 4).

Four epidemiological studies, including our previous study, have recently shown that SUA levels were closely associated with an increase in the incidence of MetS. ${ }^{11-14}$ In the present study, a different approach was used to assess the association between elevated SUA levels and the incidence of MetS. As a result, elevated SUA levels showed a significant and independent association with the incidence of MetS, even in subjects without hypertension.

The principal pathophysiological abnormalities underlying MetS are central obesity and insulin resistance. ${ }^{27}$ Therefore, subjects without MetS might have a lower degree of insulin resistance than those with MetS. SUA levels have been positively correlated with insulin resistance. ${ }^{28,29}$ We observed that WC increased with increasing SUA levels (Table 1). WC is a marker of visceral obesity, and a larger WC was correlated with a higher degree of insulin resistance. ${ }^{30}$ Visceral

Table 3 Odds ratios for the incidence of hypertension, metabolic syndrome and hypertension with metabolic syndrome according to serum uric acid quartiles

\begin{tabular}{|c|c|c|c|c|c|c|c|c|c|}
\hline \multirow[b]{2}{*}{ UA variable } & \multicolumn{3}{|c|}{$H T$} & \multicolumn{3}{|c|}{ MetS } & \multicolumn{3}{|c|}{$H T+M e t S$} \\
\hline & $O R$ & (95\% Cl) & P-values & $O R$ & $(95 \%$ Cl) & P-values & $O R$ & $(95 \%$ Cl) & P-values \\
\hline UA1 & 1.0 & Reference & - & 1.0 & Reference & - & 1.0 & Reference & - \\
\hline UA2 & 1.6 & $(1.2-2.1)$ & 0.0012 & 1.8 & $(1.3-2.6)$ & 0.0011 & 1.7 & $(0.7-4.2)$ & 0.2457 \\
\hline UA3 & 1.8 & $(1.4-2.5)$ & $<0.0001$ & 2.5 & $(1.7-3.6)$ & $<0.0001$ & 4.1 & $(1.7-9.7)$ & 0.0013 \\
\hline \multicolumn{10}{|l|}{ Model 2} \\
\hline UA1 & 1.0 & Reference & - & 1.0 & Reference & - & 1.0 & Reference & - \\
\hline UA2 & 1.5 & $(1.1-2.0)$ & 0.0129 & 1.2 & $(0.8-1.8)$ & 0.3330 & 1.3 & $(0.5-3.5)$ & 0.5503 \\
\hline UA3 & 1.5 & $(1.1-2.1)$ & 0.0128 & 1.3 & $(0.9-1.9)$ & 0.1910 & 2.7 & $(1.1-6.6)$ & 0.0276 \\
\hline UA4 & 1.8 & $(1.2-2.5)$ & 0.0022 & 1.8 & $(1.2-2.7)$ & 0.0039 & 3.2 & $(1.3-8.0)$ & 0.0115 \\
\hline
\end{tabular}

Abbreviations: $\mathrm{Cl}$, confidence interval; $\mathrm{HT}$, hypertension; $\mathrm{HT}+$ MetS, hypertension with metabolic syndrome; MetS, metabolic syndrome; OR, odds ratio; UA, uric acid.

Model 1: adjusted for age and sex.

Model 2: adjusted for age, sex, alcohol consumption, estimated glomerular filtration rate, waist circumference values and systolic blood pressure, triglyceride, high-density lipoprotein cholesterol,

low-density lipoprotein cholesterol and fasting blood glucose levels.

$\mathrm{UA1}, \leqslant 4.4 \mathrm{mg} \mathrm{dl}^{-1}$; UA2, 4.5-5.4 $\mathrm{mg} \mathrm{dl}^{-1}$; UA3, 5.5-6.4 $\mathrm{mg} \mathrm{dl}^{-1}$; and UA4, $\geqslant 6.5 \mathrm{mg} \mathrm{dl}^{-1}$.

$P<0.05$ was considered statistically significant. 
Table 4 Odds ratios for the incidence of hypertension, metabolic syndrome and hypertension with metabolic syndrome according to serum uric acid tertile or the presence of hyperuricemia

\begin{tabular}{|c|c|c|c|c|c|c|c|c|c|c|}
\hline & \multicolumn{5}{|c|}{ Males } & \multicolumn{5}{|c|}{ Females } \\
\hline & $\begin{array}{c}\text { UA1 } \\
(\mathrm{n}=846)\end{array}$ & $\begin{array}{c}\text { UA2 } \\
(\mathrm{n}=869)\end{array}$ & $\begin{array}{c}\text { UA3 } \\
(\mathrm{n}=813)\end{array}$ & $\begin{array}{c}H U(+) \\
(n=654)\end{array}$ & $\begin{array}{c}H U(-) \\
(\mathrm{n}=1874)\end{array}$ & $\begin{array}{c}\text { UA1 } \\
(n=783)\end{array}$ & $\begin{array}{c}\text { UA2 } \\
(\mathrm{n}=752)\end{array}$ & $\begin{array}{c}\text { UA3 } \\
(\mathrm{n}=749)\end{array}$ & $\begin{array}{c}H U(+) \\
(n=27)\end{array}$ & $\begin{array}{c}H U(-) \\
(n=2257)\end{array}$ \\
\hline Incidence of $\mathrm{HT}(n, \%)$ & $120(14.2)$ & $115(13.2)$ & $155(19.1)$ & $127(19.4)$ & $263(14.0)$ & $54(6.9)$ & $75(10.0)$ & $99(13.2)$ & $4(14.8)$ & 224 (9.9) \\
\hline Incidence of MetS ( $n, \%)$ & $138(16.3)$ & $212(24.4)$ & $293(36.0)$ & $247(37.8)$ & $396(21.1)$ & $19(2.4)$ & $29(3.9)$ & $73(9.7)$ & $2(7.4)$ & $119(5.3)$ \\
\hline Incidence of HT+MetS ( $n, \%)$ & $23(2.7)$ & $46(5.3)$ & $66(8.1)$ & $53(8.1)$ & $82(4.4)$ & $3(0.4)$ & $4(0.5)$ & $16(2.1)$ & $0(0)$ & $23(1.0)$ \\
\hline \multicolumn{11}{|l|}{$H T$} \\
\hline OR & 1.0 & 1.0 & 1.5 & 1.5 & & 1.0 & 1.4 & 1.8 & 1.3 & \\
\hline $95 \% \mathrm{Cl}$ & Reference & $0.7-1.3$ & $1.2-2.0$ & $1.2-1.9$ & & Reference & $1.0-2.1$ & $1.3-2.6$ & $0.4-3.8$ & \\
\hline$P$-values & - & 0.7237 & 0.0012 & 0.0004 & & - & 0.00507 & 0.0007 & 0.6429 & \\
\hline \multicolumn{11}{|l|}{ MetS } \\
\hline OR & 1.0 & 2.9 & 2.9 & 2.2 & & 1.0 & 1.6 & 4.1 & 1.2 & \\
\hline $95 \% \mathrm{Cl}$ & Reference & $1.3-2.1$ & $2.3-3.7$ & $1.8-2.7$ & & Reference & $0 . .9-2.8$ & $2.4-6.8$ & $0.3-5.3$ & \\
\hline$P$-values & - & $<0.0001$ & $<0.0001$ & $<0.0001$ & & - & 0.2024 & $<0.0001$ & 0.7812 & \\
\hline \multicolumn{11}{|l|}{$H T+M e t S$} \\
\hline OR & 1.0 & 2.0 & 3.3 & 2.0 & & 1.0 & 1.4 & 5.2 & - & \\
\hline $95 \% \mathrm{Cl}$ & Reference & $1.2-3.4$ & $2.1-5.4$ & $1.4-2.8$ & & Reference & $0.3-6.1$ & $1.5-18.2$ & - & \\
\hline$P$-values & - & 0.0060 & $<0.0001$ & 0.0002 & & - & 0.6932 & 0.0091 & - & \\
\hline \multicolumn{11}{|l|}{$H T$} \\
\hline OR & 1.0 & 0.8 & 1.3 & 1.5 & & 1.0 & 1.5 & 1.5 & 0.5 & \\
\hline $95 \% \mathrm{Cl}$ & Reference & $0.6-1.1$ & $1.0-1.8$ & $1.1-1.9$ & & Reference & $1.0-2.2$ & $1.0-2.2$ & $0.2-1.7$ & \\
\hline$P$-values & - & 0.2652 & 0.0562 & 0.0057 & & - & 0.0696 & 0.0590 & 0.2858 & \\
\hline \multicolumn{11}{|l|}{ MetS } \\
\hline OR & 1.0 & 1.3 & 1.6 & 1.4 & & 1.0 & 1.2 & 1.5 & 0.3 & \\
\hline $95 \% \mathrm{Cl}$ & Reference & $1.0-1.7$ & $1.2-2.1$ & $1.1-1.7$ & & Reference & $0.6-2.2$ & $0.8-2.8$ & $0.1-1.6$ & \\
\hline$P$-values & - & 0.0882 & 0.0010 & 0.0088 & & - & 0.6311 & 0.1714 & 0.1470 & \\
\hline \multicolumn{11}{|l|}{$H T+M e t S$} \\
\hline OR & 1.0 & 1.8 & 2.4 & 1.5 & & 1.0 & 1.1 & 1.9 & - & \\
\hline $95 \% \mathrm{Cl}$ & Reference & $1.0-3.1$ & $1.4-4.2$ & $1.0-2.3$ & & Reference & $0.2-5.0$ & $0.5-7.4$ & - & \\
\hline$P$-values & - & 0.0381 & 0.0015 & 0.0403 & & - & 0.9121 & 0.3380 & - & \\
\hline
\end{tabular}

Abbreviations: $\mathrm{Cl}$, confidence interval; HT, hypertension; HT+MetS, hypertension with metabolic syndrome; MetS, metabolic syndrome; OR, odds ratio; UA, uric acid.

Model 1: adjusted for age.

Model 2: adjusted for age, alcohol consumption, estimated glomerular filtration rate, waist circumference values and systolic blood pressure, triglyceride, high-density lipoprotein cholesterol,

low-density lipoprotein cholesterol and fasting blood glucose levels.

$\mathrm{UA1}, \leqslant 5.8 \mathrm{mg} \mathrm{dl}^{-1}$; UA2, 5.9-6.8 $\mathrm{mg} \mathrm{dl}^{-1}$; and $\cup A 3, \geqslant 6.9 \mathrm{mg} \mathrm{dl}^{-1}$ for males.

$\mathrm{UA1}, \leqslant 4.1 \mathrm{mg} \mathrm{dl}^{-1}$; UA2, $4.2-4.9 \mathrm{mg} \mathrm{dl}^{-1}$; and $\cup A 3, \geqslant 5.0 \mathrm{mg} \mathrm{dl}^{-1}$ for females.

Hyperuricemia was defined as serum uric acid levels of $>7.0 \mathrm{mg} \mathrm{dl}^{-1}$.

The value of $P<0.05$ was considered statistically significant.

adipose tissues secrete several adipocytokines, including adiponectin, leptin and tumor necrosis factor-1. Elevated production of these proinflammatory adipocytokines contributes to the development of insulin resistance. ${ }^{31}$ Moreover, evidence that elevated SUA levels increase the risk of developing insulin resistance has been reported. ${ }^{32}$

In the present study, significant associations between elevated SUA levels and the incidences of hypertension and MetS were determined using a multivariate adjusted model that included WC. Therefore, other contributing factors such as endothelial dysfunction and oxidative stress are also involved in the pathophysiological mechanisms leading to elevated SUA levels and the incidences of hypertension and MetS. ${ }^{16-18}$ UA potently decreases endothelial nitric oxide bioavailability and induces endothelial dysfunction. ${ }^{33}$ Elevated SUA levels were also associated with the increased generation of free radicals ${ }^{34}$ and oxidative stress, which could abolish endothelium-dependent vasodilatation, leading to hypertension. ${ }^{35}$ However, other studies have suggested that UA was an effective antioxidant, ${ }^{36,37}$ and the exact role of UA in oxidation (pro-oxidant or antioxidant) remains to be elucidated. Further studies are needed to clarify the role of UA in the context of hypertension and MetS.

The present study had several limitations. First, we showed significant associations between elevated SUA levels and the incidences of hypertension and MetS; however, no causal relationship could be inferred because this study was observational. In addition, although insulin resistance, endothelial dysfunction, oxidative stress and inflammation might have roles in the incidences of hypertension and MetS, epidemiological studies such as ours cannot directly demonstrate pathophysiological evidence. Second, variables were measured only once; therefore, misclassifications might have occurred. A lack of data regarding the use of antihyperuricemic medications might have led to underestimation of the prevalence of hyperuricemia. Because the frequency of diuretics used as antihypertensives is low in Japan, ${ }^{38}$ the influence of diuretics on our results was most likely small. Third, information on menopause was not available for our female subjects. Estrogen increases the excretion of UA, and SUA levels increase after 
menopause. ${ }^{39}$ The biological mechanism explaining the relationships between estrogen levels and the development of hypertension or MetS has still not been elucidated. Fourth, we could not assess the dietary statuses of our subjects. Finally, the subjects in this study participated voluntarily and represented a relatively healthy population, and this voluntary participation might have led to selection bias. Despite these limitations, our results offer new insight into the relationships between SUA levels and the incidences of hypertension and MetS.

In conclusion, increased SUA levels were significantly and independently associated with the incidences of hypertension and MetS in subjects without hypertension or MetS at baseline. Increased SUA levels might also be associated with the incidence of hypertension with MetS. To prevent cardiovascular events, this relationship between increased SUA levels and an increased incidence of hypertension and MetS should be noted, even in subjects without hypertension or MetS at baseline.

\section{CONFLICT OF INTEREST}

The authors declare no conflict of interest.

\section{ACKNOWLEDGEMENTS}

We thank the staff of the Okinawa General Health Maintenance Association for their cooperation.

Author contributions: $\mathrm{K}$ Kinjo designed the data collection, $\mathrm{T}$ Inoue and $\mathrm{K}$ Kohagura provided valuable assistance, and Y Ohya co-wrote the paper.

1 Cannon PJ, Stason WB, Demartini FE, Sommers SC, Laragh JH. Hyperuricemia in primary and renal hypertension. New Engl J Med 1966; 275: 457-464.

2 Schmidt MI, Watson RL, Duncan BB, Metcalf P, Brancati FL, Sharrett AR, Davis CE, Heiss G. Clustering of dyslipidemia, hyperuricemia, diabetes, and hypertension and its association with fasting insulin and central and overall obesity in a general population: Atherosclerosis Risk in Communities Study Investigators. Metabolism 1996; 45 699-706.

3 Feig DI, Kang DH, Johnson RJ. Uric acid and cardiovascular risk. New Engl J Med 2008; 359: 1811-1821.

4 Choi HK, Ford ES. Prevalence of the metabolic syndrome in individuals with hyperuricemia. Am J Med 2007; 120: 442-447.

5 Tsouli SG, Liberopoulos EN, Mikhailidis DP, Athyros VG, Elisaf MS. Elevated serum uric acid levels in metabolic syndrome: an active component or an innocent bystander? Metabolism 2006; 55: 1293-1301.

6 Yoo TW, Sung KC, Shin HS, Kim BJ, Kim BS, Kang JH, Lee MH, Park JR, Kim H Rhee EJ, Lee WY, Kim SW, Ryu SH, Keum DG. Relationship between serum uric acid concentration and insulin resistance and metabolic syndrome. Circ J 2005; 69: 928-933.

7 Nagahama K, Iseki K, Inoue T, Touma T, Ikemiya Y, Takishita S. Hyperuricemia and cardiovascular risk factor clustering in a screened cohort in Okinawa, Japan. Hypertens Res 2004; 27: 227-233.

8 Nagahama K, Inoue T, Iseki K, Touma T, Kinjo K, Ohya Y, Takishita S. Hyperuricemia as a predictor of hypertension in a screened cohort in Okinawa, Japan. Hypertens Res 2004; 27: 835-841.

9 Perlstein TS, Gumieniak O, Williams GH, Sparrow D, Vokonas PS, Gaziano M, Weiss ST, Litonjua AA. Uric acid and the development of hypertension-The Normative Aging Study. Hypertension 2006; 48: 1031-1036.

10 Krishnan E, Kwoh CK, Schumacher HR, Kuller L. Hyperuricemia and incidence of hypertension among men without metabolic syndrome. Hypertension 2007; 49 298-303.

11 Nagahama K, Inoue T, Kohagura K, Ishihara A, Kinjo K, Ohya Y. Hyperuricemia predicts future metabolic syndrome: a 4-year follow-up study of a large screened cohort in Okinawa, Japan. Hypertens Res 2014; 37: 232-238.

12 Sui X, Church TS, Meriwether RA, Lobelo F, Blair SN. Uric acid and the development of metabolic syndrome in women and men. Metabolism 2008; 57: 845-852.

13 Hara S, Tsuji H, Ohmoto Y, Amakawa K, Hsieh SD, Arase Y, Nakajima H. High serum uric acid level and low urine $\mathrm{pH}$ as predictors of metabolic syndrome: a retrospective cohort study in a Japanese urban population. Metabolism 2011: 24: 281-288.
14 Yang T, Chu CH, Bai CH, You SL, Chou YC, Chou WY, Chien KL, Hwang LC, Su TC Tseng $\mathrm{CH}$, Sun $\mathrm{CA}$. Uric acid level as a risk marker for metabolic syndrome: a Chinese cohort study. Atherosclerosis 2012; 220: 525-531.

15 Johnson RJ, Kang DH, Feig D, Kivlighn S, Kanellis J, Watanabe S, Tuttle KR Rodriguez-Iturbe B, Herrera-Acosta J, Mazzali M. Is there a pathogenetic role for uric acid in hypertension and cardiovascular and renal disease? Hypertension 2003; 41 1183-1190.

16 Li C, Hsieh MC, Chang SJ. Metabolic syndrome, diabetes, and hyperuricemia. Curr Opin Rheumatol 2013; 25: 210-216.

17 Edwards NL. The role of hyperuricemia in vascular disease. Curr Opin Rheumatol 2009; 21: 132-137.

18 So A, Thorens B. Uric acid transport and disease. J Clin Invest 2010; 120 1791-1799.

19 Ferrannini E, Buzzigoli G, Bonadonna R, Giorico MA, Oleggini M, Graziadei L, Pedrinelli R, Brandi L, Bevilacqua S. Insulin resistance in essential hypertension. New Engl J Med 1987; 317: 350-357.

20 Swislocki AL, Hoffman BB, Reaven GM. Insulin resistance, glucose intolerance and hyperinsulinemia in patients with hypertension. Am J Hypertens 1989; 2: 419-423.

21 Pollare $\mathrm{T}$, Lithell $\mathrm{H}$, Berne $\mathrm{C}$. Insulin resistance is a characteristic feature of primary hypertension independent of obesity. Metabolism 1990; 39: 167-174.

22 Arcucci O, de Simone G, Izzo R, Rozza F, Chinali M, Rao MA, Bodenizza C, De Luca N Trimarco B. Association of suboptimal blood pressure control with body size and metabolic abnormalities. J Hypertens 2007; 25: 2296-2300.

23 Ninomiya T, Kubo M, Doi Y, Yonemoto K, Tanizaki Y, Rahman M, Arima H, Tsuryuya K, lida M, Kiyohara Y. Impact of metabolic syndrome on the development of cardiovascular disease in a general Japanese population: the Hisayama study. Stroke 2007; 38: 2063-2069.

24 Guideline Revising Committee of Japanese Society of Gout and Nucleic Acid Metabolism, ed. Revised Guideline for the Management of Hyperuriceima and Gout. Osaka: Medical Review Co Ltd 2010.

25 Matsuo S, Imai E, Horio M, Yasuda Y, Tomita K, Nitta K, Yamagata K, Tomino Y, Yokoyama $\mathrm{H}$, Hishida A. Collaborators developing the Japanese equation for estimated GFR. Revised equations for estimated GFR from serum creatinine in Japan. Am J Kidney Dis 2009; 53: 982-992.

26 The Japanese Committee on the Criteria for MetS. Definition and the diagnostic standard for metabolic syndrome - Committee to Evaluate Diagnostic Standards for Metabolic Syndrome. Nippon Naika Gakkai Zassi 2005; 94: 749-809.

27 Kahn R, Buse J, Ferrannini E, Stern M. American Diabetes Association; European Association for the Study of Diabetes. The metabolic syndrome: time for a critical appraisal: joint statement from the American Diabetes Association and the European Association for the Study of Diabetes, Diabetes Care 2005; 28 2289-2304.

28 Vuorinen-Markkola H, Yki-Järvinen H. Hyperuricemia and insulin resistance. J Clin Endocrinol Metab 1994; 78: 25-29.

29 Modan M, Halkin H, Karasik A, Lusky A. Elevated serum uric acid-a facet of hyperinsulinaemia. Diabetologia 1987; 30: 713-718.

30 Reaven GM. The metabolic syndrome: time to get off the merry-go-round? J Intern Med 2011; 269: 127-136.

31 Bruce KD, Byrne CD. The metabolic syndrome: common origins of a multifactorial disorder. Postgrad Med J 2009; 85: 614-621.

32 Krishnan E, Pandya BJ, Chung L, Hariri A, Dabbous O. Hyperuricemia in young adults and risk of insulin resistance, prediabetes, and diabetes: a 15-year follow-up study. Am J Epidemol 2012; 176: 108-116.

33 Khosla UM, Zharikov S, Finch JL, Nakagawa T, Roncal C, Mu W, Krotova K, Block ER, Prabhakar S, Johnson RJ. Hyperuricemia induces endothelial dysfunciton. Kidney Int 2005; 67: 1739-1742.

34 Vasquez-Vivar J, Santos AM, Junqueria VB, Augusto O. Peroxynitrite-mediated formation of free radicals in human plasma: EPR detection of ascorbyl, albuminthiyl and uric acidderived free radicals. Biochem J 1996; 314: 869-876.

35 Auch-Schwelk W, Katusic ZS, Vanhoutte PM. Contractions to oxygen-derived free radicals are augmented in aorta of the spontaneously hypertensive rat. Hypertension 1989; 13: 859-864.

36 Nieto FJ, Iribarren C, Gross MD, Comstock GW, Cutler RG. Uric acid and serum antioxidant capacity: a reaction to atherosclerosis? Atherosclerosis 2000; 148 131-139.

37 Nyyssonen K, Porkkala-Sarataho E, Kaikkonen J. Ascorbate and urate are the strongest determinants of plasma antioxidative capacity and serum lipid resistance to oxidation in Finish men. Atherosclerosis 1997; 130: 223-233.

38 Yamamoto Y, Sonoyama K, Matsubara K, Furuse M, Yatsuhashi T, Hamada T, Ogino K, Igawa $\mathrm{O}$, Hisatome I, Shigemasa $\mathrm{C}$. The status of hypertension management in Japan in 2000. Hypertens Res 2002; 25: 717-725.

39 Yahyaoui R, Esteva I, Haro-Mora JJ, Almaraz MC, Morcillo S, Rojo-Marti'nez G, Marti'nez J, Go'mez-Zumaquero JM, Gonza'lez I, Hernando V, Soriguer F. Effect of long-term administration of cross-sex hormone therapy on serum and urinary uric acid in transsexual persons. J Clin Endocrinol Metab 2008. 93: 2230-2233. 